\title{
The collection of electron diffraction intensity data and their use in structure determination
}

\author{
J. Gjonnes* , V. Hansen ${ }^{* *}$ and X.Z. Li ${ }^{* * *}$ \\ *Center for Materials Science, University of Oslo, Gaustadalleen 21 N-0349 Oslo, Norway \\ ** Stavanger University College, Departement of Technology and Natural Sciences, \\ P.O. Box 2557 Ullandhaug N-4091 Stavanger, Norway \\ **** Walter Scott Engineering Center, University of Nebraska, Lincoln, NE 68588-0656. USA
}

Electron diffraction crystallography has re-emerged in recent years, as a realistic option for structure solution, by itself or in combination with high-resolution imaging (HRTEM). This is connected with several developments, of improved detection (imaging plates, SSCCD, energy filter), of new measuring concepts, and better crystallographic procedures. Further progress in the field will depend on the extent and precision of intensity data that can be collected. We shall discuss three principles for collecting electron diffraction intensities:

1) the usual way - the SAED or microdiffraction spot pattern, collected with a stationary beam along a zone axis works best for thin crystals in dense zones. Structures have been solved mainly in projection, often in combination with HRTEM [1,2,3]; satisfactory refinement may be obtained when dynamical scattering is included.

2) the precise way - the CBED-profile, or high-resolution electron diffraction (in analogy with current $\mathrm{X}$-ray terminology) has been developed for precise refinement of small-unit cell structure factors. The technique can also be used $a b$ initio for unknown structures, including accurate structure factor phases in the non-centrosymmetrical case $[4,5]$.

3) the X-ray way - integrating intensities through the Bragg angle. In electron diffraction this can be achieved with the aid of scanning systems, or by integrating across features in CBED patterns. The precession technique [6,7] emulates the precession photograph in X-ray diffraction: the incident beam is tilted off a zone axis and precessed around this, combined with de-scanning of the pattern below the specimen. A Laue circle of reflections is swept through the zero Laue zone, Fig 1; a spot pattern similar to SAED is obtained. There are several advantages: sensitivity to variations in local thickness and orientation is reduced. Effects of dynamical scattering are reduced, especially those involving non-systematic interactions. This is important when several patterns are merged by normalization to common rows of reflections into a three-dimensional data set. The data can extend to high scattering angle, crystals need not be very thin.

The three-dimensional data can be treated as kinematical in the early stages of a structure determination, for calculations of Patterson projections and sections, or in direct method programs. All atoms may not be located, the structure can be completed by chemical considerations and/or by Fourier refinement, and confirmed by least squares refinement. The fit may be poor, but the general experience is that atomic arrangement tend to be correct. Improvement of the fit must be based on dynamical calculations. A confirmation/refinement strategy can utilize approximate, two-beam like expressions for integrated intensities in off-axis configurations: 


$$
\int I_{g}^{\text {two-beam }}\left(s_{g}, t\right) d s_{g}=U_{g} \int_{0}^{U_{g} t} J_{0}(x) d x
$$

where $s_{g}$ is the excitation error, $t$ thickness, $U_{g}$ the Fourier potential and $J_{0}$ the zero order Bessel function. This can be extended to cover multiple beam cases by introduction of dynamical or effective potentials - assuming two-beam like profile, and suitable approximations for the $U_{g}^{\text {eff }}$, e.g. the Bethe potentials: $U_{g}{ }^{e f f}=U_{g}-\Sigma_{h} U_{h} U_{g-h} / 2 k s_{h}$, where the excitation errors $s_{h}$ refer to the position at the precession circle, i.e. $s_{g}=0$. By combining these two expressions in a statistical procedure, applicable to the centrosymmetric case, we can retrieve $U_{g}$, (with signs) in a projection from experimentally determine integrated intensities, as described in [8]. The result can be used for calculation of difference Fourier maps, or a least squares refinement. Application to aluminum alloy system will be referred, including combiation with high-resolution diffraction (CBED).

Coherent precipitates are common in alloy system. When these are large enough, the incident beam can be focussed on a single precipitate extending through the thickness. In other cases, as in the Al$\mathrm{Zn}-\mathrm{Mg}$ alloys, the coherent metastable are smaller and embedded in the Al matrix. SAED diffraction patterns are superpositions of four orientations, with extensive overlap of reflections. Measurement is a compromise between low-order matrix zones, with strong multiple diffraction, and projections, with fewer reflections and contributions from upper layers. Structure model has been proposed from HRTEM images.

\section{References}

[1] H.W. Zandbergen, et al. Micron 30 (1999) 395

[2] I.G. Voigt-Martin, et al. Acta Cryst A56 (2000) 436

[3] D.L. Dorset, Structural Electron Crystallography. Plenum, New York, 1995

[4] Y.F.Cheng, et al. Acta Cryst. A52 (1996) 923

[5] R. Høier, et al. Acta Cryst. A55(1999) 188

[6] R.Vincent and P Midgley, Ultramicroscopy 53 (1994) 271

[7] E. Sørbrøden et al, Proc ICEM 14 Cancun, Mexico 1998. Vol I, 425

[8] K. Gjønnes, et al. Acta cryst A54 (1998)102
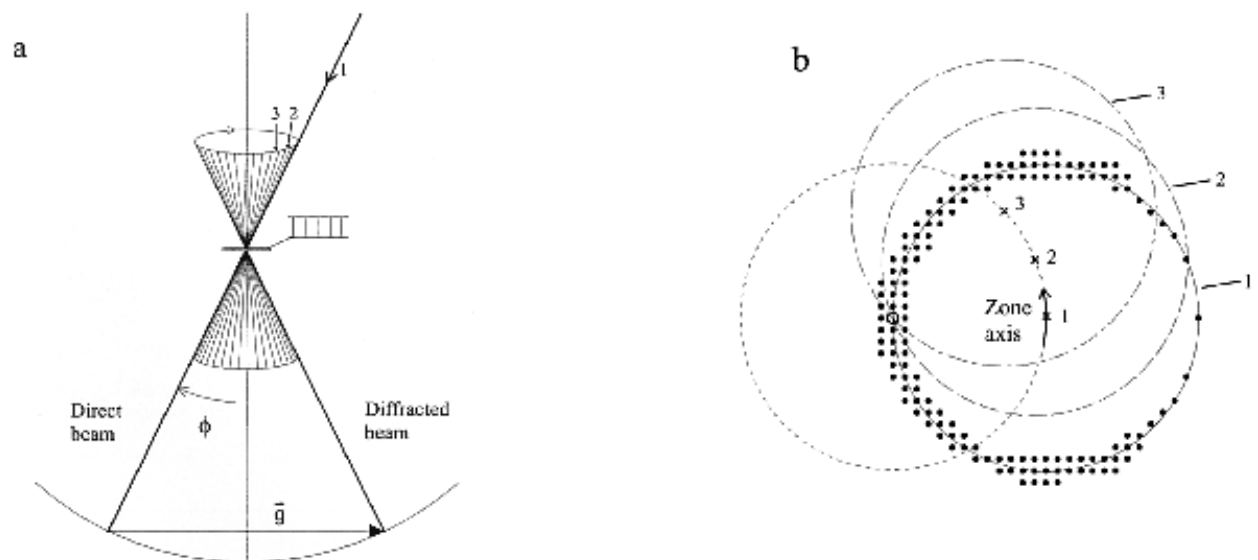

Fig. 1a Precession principle, b) diffraction conditions: Laue circles. 